\title{
Impact of Branching on the Phase Behavior of Polymers
}

\author{
Gaurav Arya* and Athanassios Z. Panagiotopoulos \\ Department of Chemical Engineering, Princeton University, Princeton, New Jersey 08544
}

Received July 14, 2005; Revised Manuscript Received September 20, 2005

\begin{abstract}
We have studied the effect of branching on the solution phase behavior of branched homopolymers using grand canonical Monte Carlo (GCMC) simulations in conjunction with multihistogram reweighting and finite-size scaling analysis. The critical temperature $\left(T_{\mathrm{c}}\right)$ and the $\Theta$ temperature $(\Theta)$ decrease as polymer branching is increased, but the drop in $\Theta$ is less pronounced than that of $T_{\mathrm{c}}$. The critical volume fraction $\left(\phi_{\mathrm{c}}\right)$ rises with the degree of branching. Branched polymers are found to obey the Shultz-Flory relationship and exhibit a power-law behavior in $\phi_{\mathrm{c}}$ vs chain length, with similar scaling exponents as those for their linear counterparts. Comparisons of the GCMC results are made to results of the lattice cluster theory (LCT). It is observed that the LCT significantly underestimates the impact of polymer branching on the critical behavior of polymers. We speculate this discrepancy between the two formulations to be due to an inadequate representation of the variation of polymer conformations with branching and neglect of fluctuations in the LCT theory.
\end{abstract}

\section{Introduction}

Branched and hyperbranched polymers (dendrimers) have received a lot of attention recently due to their emerging applications in catalysis, ${ }^{1}$ nanomaterial synthesis ${ }^{2}$ and biomedicine. ${ }^{3,4}$ Understanding the role that branching plays in determining the rheological and thermodynamical properties of a polymer melt or its solution is currently an active area of research. Although significant advancements have been made in elucidating the conformational properties of branched polymers, ${ }^{5}$ comparatively little progress has been made in understanding the impact of branching on the miscibility and critical behavior of polymers in solution. In this study, we investigate the effect of branching on the liquidliquid phase separation and critical parameters of polymer solutions using Monte Carlo simulations.

Within a certain polymer concentration range, a polymer-poor solvent solution phase separates into a polymer-lean and a polymer-rich phase to minimize its overall free energy. Such a phase separation leads to a reduction both in the number of enthalpic interactions between the polymer and the solvent and in the mixing entropy. The mixing entropy and enthalpy are complex functions of the polymer structure. Intuitively, one expects that a branched polymer will display fewer unfavorable polymer-solvent interactions than a linear polymer with an identical molecular weight. This would imply that the branched polymers should exhibit an increased miscibility and lower upper critical solution temperatures as compared to linear polymers. Recent experiments involving measurements of the cloud points of linear and star-branched polystyrene polymers in a methylcyclohexane solvent show a drop in the critical temperature $\left(T_{\mathrm{c}}\right)$ as the degree of branching increases, a trend consistent with the above heuristic argument. ${ }^{6-8}$ From the viewpoint that branched polymers are generally more compact that their linear counterparts, we expect that the critical volume fraction $\left(\phi_{c}\right)$ of branched polymers should exceed that of the linear polymers with similar molecular weights. As far as experiments are

* Corresponding author. E-mail: garya@nyu.edu; Current address: Department of Chemistry, New York University, New York, NY 10003. concerned, contrasting observations have been made regarding the dependence of $\phi_{c}$ on branching, with some experiments showing an increase in $\phi_{\mathrm{c}}$ with branching, ${ }^{6}$ while others showing no discernible effect of branching on $\phi_{\mathrm{c}}{ }^{8}$

Several theoretical approaches have been proposed to model the effect of branching on $T_{\mathrm{c}}$ and $\phi_{\mathrm{c}}$. It is wellknown that the classic Flory-Huggins theory ${ }^{9}$ does not capture the effect of branching on polymer phase separation. The polymer theories which do capture the effect of branching include a scaling theory developed by Daoud et al., ${ }^{10}$ a theory developed by Saeki, ${ }^{11}$ which replaces the standard mixing entropy term of FloryHuggins with a combinatorial entropy term more applicable to star polymers, and the lattice cluster theory due to Freed and co-workers. ${ }^{12}$ All these theories predict a drop in $T_{\mathrm{c}}$ and a small rise in $\phi_{\mathrm{c}}$ as a polymer becomes more branched. However, the magnitude of these trends as well as predictions of $T_{\mathrm{c}}$ and $\phi_{\mathrm{c}}$ have yet to be properly compared with experimental results. Another drawback of these theories is that they are all based on a meanfield approximation which neglects the effects of fluctuations. The predictions for $T_{\mathrm{c}}$ and $\phi_{\mathrm{c}}$ through these theories have therefore often been inaccurate. To the best of our knowledge, there does not presently exist any renormalization theory which properly incorporates both the effect of fluctuations and branching on the phase behavior of polymers. A majority of Monte Carlo simulations, which naturally incorporate the effect of fluctuations, have focused more on the conformational properties of polymers rather their critical and phase behavior. ${ }^{13-18}$ The remaining Monte Carlo studies have investigated the phase behavior of a very restricted subset of isomers of short alkanes. ${ }^{19,20}$ Making concrete conclusions about the role of branching from these studies has been impossible. Hence, in short, our understanding of the effect of branching on the phase behavior of branched polymers remains rather poor.

In this paper, we use extensive grand canonical Monte Carlo (GCMC) simulations in conjunction with multihistogram reweighting and finite size scaling analysis to obtain the phase coexistence envelopes and the critical points of lattice polymers with varying degrees of branching. Subsequently, we are able to test the accuracy of the lattice cluster theory, which is the most 

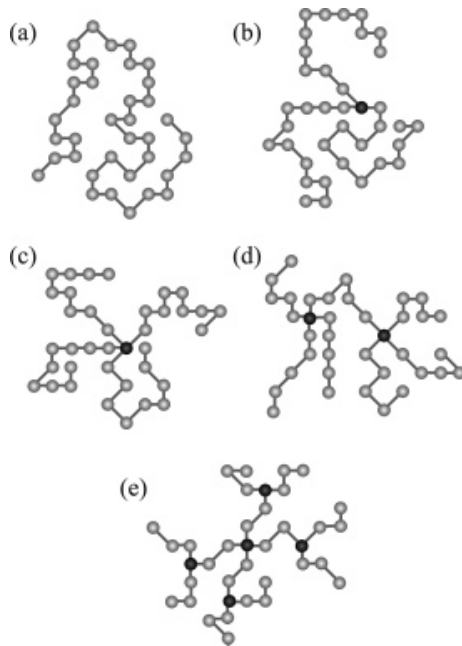

Figure 1. Schematic of the five polymer structures considered in this study: (a) linear (L), (b) single branch (B1), (c) two branches (B2), and (d) four branches (B4), and (e) dendrimer (D) for $r=37$. The dark colored beads represent branching points.

sophisticated of the three theories mentioned above, and properly evaluate the role of fluctuations on the phase behavior of branched polymers. Another objective of this study has been to evaluate the scaling behavior of the critical $\left(T_{\mathrm{c}}\right.$ and $\left.\phi_{\mathrm{c}}\right)$ and conformational parameters (polymer radii of gyration) of branched polymers with respect to their polymerization index, and compare them with those obtained for linear polymers.

\section{Model and Simulations Methods}

Our system is composed of a branched lattice homopolymer with a polymerization index $r$ on a cubic lattice with a coordination number $z=26$. In this study, $r$ is varied within the range $r=65$ to $r=389$. The linear portions of the polymer are modeled as a series of connected nearest-neighbor lattice sites, while the branch points consist of a single site connected to more than two linear portions of the polymer. The five different polymer architectures studied consist of a linear chain (L), lightly branched polymers with one (B1), two (B2), and four branches (B4), and a highly branched second generation dendrimer (D). We ensure that the length of the free ends of the branches and the length of the separators between two neighboring branch points are kept equal for consistency except for round-off of noninteger chain lengths. Figure 1 gives schematic illustrations of the polymer structures studied. Solvent molecules occupy all lattice sites not excluded by the polymer.

Nearest neighbor polymer and solvent sites interact with each other through the energy parameters $\epsilon_{\mathrm{pp}}, \epsilon_{\mathrm{ps}}$, and $\epsilon_{\mathrm{sS}}$. Because of lattice coordination constraints, there is a single relevant energy parameter $\epsilon$ defined as

$$
\epsilon=2 \epsilon_{\mathrm{ps}}-\epsilon_{\mathrm{pp}}-\epsilon_{\mathrm{ss}}
$$

The reduced temperature is then given by

$$
T^{*}=\frac{k_{\mathrm{B}} T}{\epsilon}
$$

where $k_{\mathrm{B}}$ is the Boltzmann's constant. For convenience, the asterisk superscript will be omitted from the reduced temperature henceforth. A cubic simulation box of length $L$ along with periodic boundary conditions along the three coordination axes is employed. The polymer volume fraction $\phi$ is therefore defined as

$$
\phi=\frac{r N}{L^{3}}
$$

where $N$ is the number of polymer chains within the simulation box.

Our GCMC simulations are conducted at a constant chemical potential of $\mu$, temperature $T$ and volume $V=$ $L^{3}$. The polymer chains are inserted and deleted according to the configurational bias method ${ }^{21}$ with acceptance criteria given by

$$
\begin{gathered}
P_{\text {ins }}=\min \left[1, \frac{1}{W} \frac{V}{N+1} \exp (-\beta \Delta U+\beta \mu)\right] \\
P_{\text {del }}=\min \left[1, \frac{W N}{V} \exp (-\beta \Delta U-\beta \mu)\right]
\end{gathered}
$$

where $\beta=1 / k_{\mathrm{B}} T, \Delta U$ is the internal energy change of the system upon inserting or deleting a polymer, while $W$ is the magnitude of the bias (weight) applied in generating polymer conformations using the Rosenbluth procedure. ${ }^{22,23}$ For enhanced sampling of the phase space, we also allow for translation of polymer chains and regrowth of individual polymer branches. The acceptance criterion for the translational moves is

$$
P_{\text {trans }}=\min [1, \exp (-\beta \Delta U)]
$$

where $\Delta U$ is the internal energy change of the system upon translation of a polymer chain, while the acceptance criterion for regrowth moves is

$$
P_{\text {regrow }}=\min \left[1, \frac{W_{\text {del }}}{W_{\text {grow }}} \exp (-\beta \Delta U)\right]
$$

where $W_{\text {del }}$ and $W_{\text {grow }}$ refer to the Rosenbluth weights corresponding to deleting a polymer branch and regrowing it, respectively.

The simulations yield a histogram $f_{\mu_{i}, \beta_{i}}(N, U)$ of the frequency of occurrence of $N$ polymer chains with a total internal energy $U$. The distribution $f_{\mu, \beta}(N, U)$ is formally described by

$$
f_{\mu, \beta}(N, U)=\Omega(N, V, U) \exp (\beta(\mu N-U)) / \Xi(\mu, V, T)
$$

where $\Omega(N, V, U)$ is the microcanonical partition function (density of states) and $\Xi(\mu, V, T)$ is the grand canonical partition function. Using eq 8 , it can be shown that a histogram at a set of conditions $\mu$ and $\beta$ may be used to predict the histogram at any other condition $\mu^{\prime}$ and $\beta^{\prime}$ using the relation

$$
\frac{f_{\mu^{\prime}, \beta^{\prime}}(N, U)}{f_{\mu, \beta}(N, U)} \propto \exp \left[\left(\beta^{\prime} \mu^{\prime}-\beta \mu\right) N-\left(\beta^{\prime}-\beta\right) U\right]
$$

However, such an extrapolation is not practical far from the original $\beta$ and $\mu$ as the statistical accuracy of the histograms becomes low away from their peak value. Because of this, one needs to generate several histograms at different sets of conditions $\mu$ and $\beta$ and then combine them using a suitable procedure. In this study, the simulations were performed at values of $\mu$ and $\beta$ near the anticipated critical point. The different histograms were then combined using the procedure of 
Ferrenberg and Swendsen ${ }^{24-26}$ to generate a "universal" histogram applicable to a broader range of $\mu$ and $\beta$ given by

$$
P_{\mu, \beta}(N, U)=\frac{\left.\sum_{i=1}^{N_{\mathrm{h}}} f_{\mu_{i}, \beta_{i}}(N, U) \exp (-\beta \mathrm{U}+\beta \mu N)\right)}{\sum_{i=1}^{N_{\mathrm{h}}} K_{i} \exp \left(-\beta_{i} U+\beta_{i} \mu_{i} N-C_{i}\right)}
$$

where $N_{\mathrm{h}}$ represents the total number of histograms being combined, $K_{i}$ represents the total number of observations in run $i$, and $C_{i}$ represents a set of constants determined by solving the following set of equations iteratively with eq 10 :

$$
\exp \left(C_{i}\right)=\sum_{N} \sum_{U} P_{\mu_{i} \beta_{i}}(N, U)
$$

The final step, according to the mixed-field finite-size scaling theory, ${ }^{27,28}$ requires one to map the combined distribution (histogram) $P_{\mu, \beta}(U, N)$ to a distribution in the ordering operator $\mathbb{M}$ given by

$$
\mathscr{M}=N-\tilde{s} U
$$

where $\tilde{s}$ is a field-mixing parameter which controls the strength of coupling between the energy and density fluctuations near the critical point. At the critical point, the probability distribution, $P_{L}(x)$, should follow a universal distribution corresponding to Ising-type criticality with short-range interactions. The scaling parameter $x$ is given by

$$
x=a(L, \tilde{r})\left(\mathscr{M}-\mathscr{W}_{c}\right)
$$

where $a(L, \tilde{r})$ is a nonuniversal parameter dependent on the system size $L$ and $\tilde{r}$, which is a system-specific quantity controlling the degree of field mixing. $\mathscr{M}_{c}$ is the value of $\mathscr{T}$ at the "guessed" critical point. The quantity $a(L, \tilde{r})$ is chosen such that a unit variance is observed in the distribution $P_{L}(x)$. Hence, the procedure for obtaining the critical point involves tweaking the parameters $\mu, \beta$, and $\tilde{s}$ in eqs $10-13-$ which changes both the value of $\mathscr{N}_{c}$ as well as the distribution of $\mathscr{M}_{\text {about }}$ it-until the best match between $P_{L}(x)$ and the ideal Ising distribution is obtained. The values of $\mu$ and $T$ thus obtained represent the apparent critical point $\mu_{\mathrm{c}}$ and $T_{\mathrm{c}}$ respectively. The critical volume faction, $\phi_{\mathrm{c}}$, is obtained as the weighted average of $\phi$ with the distribution $P_{\mu, \beta}(N, U)$ at the critical point, as given by

$$
\phi_{\mathrm{c}}=\frac{r}{V} \frac{\sum_{N} \sum_{U} N \times P_{\mu_{\mathrm{c}} \beta_{\mathrm{c}}}(N, U)}{\sum_{N} \sum_{U} P_{\mu_{\mathrm{c}} \beta_{\mathrm{c}}}(N, U)}
$$

It is well-established that the critical points obtained using this procedure are power-law functions of the system size $L .{ }^{28}$ Since only the critical points obtained in the infinite system-size limit (i.e., $L \rightarrow \infty$ ) are physically meaningful, one is expected to extrapolate the critical points obtained at finite system sizes to the infinite system size limit. However, such an extrapolation was not utilized in the present study, as the finitesize corrections to the chemical potential for comparable systems were found to be small. ${ }^{29}$ It should be noted that the simulation box sizes employed in this work were consistently 5-10 times larger than the radii of gyration of the polymers. All critical data presented in this study hence correspond to reported finite system sizes only.

The procedure for obtaining accurate critical points involves running several short exploratory simulations ( $\sim 0.1-1$ million $\mathrm{MC}$ steps) at varying conditions of $\mu$ and $T$ to pinpoint the approximate location of the critical point. Frequent jumps in the density (polymer volume fraction) as well as the presence of a steep densitychemical potential curve are the most visible signatures of an approaching critical point. Histograms from 5 to 10 medium-range simulation runs $(\sim 50-100$ million MC steps) in the vicinity of the approximated critical region are then combined and used along with the mixed-field finite-size scaling analysis to yield preliminary estimates of the critical point. Finally, one to three very long simulation runs $(\sim 200-1000$ million MC steps) are performed to obtain accurate estimates of the critical point.

"Two-phase" transitions in our simulations are signified by the appearance of two peaks in the combined histogram $P_{\mu, \beta}(U, N)$ corresponding to the polymer-lean and polymer-rich phases. The conditions for phase coexistence across the two phases are equality of temperature, chemical potential and pressure (osmotic pressure of the polymer chains). The first two criteria are satisfied by construct. The pressure of a phase $(\mathscr{Q})$ may be computed to within a multiplicative constant using the relation

$$
\sum_{N} \sum_{U} P_{\mu_{i}, \beta_{i}}(N, U) \propto \ln \Xi\left(\mu_{i}, V, T_{i}\right)=\beta_{i} \mathscr{P}_{i} V
$$

To impose the third criterion, one must therefore equate the integral of the histogram $P_{\mu, \beta}(N, U)$ corresponding to the two phases in $P_{\mu, \beta}(N, U)$. The coexistence densities for a subcritical temperature $T<T_{\mathrm{c}}$ are thus obtained by reweighting the histogram (i.e., by manipulating $\mu$ ) until the two peaks in $P_{\mu, \beta}(N, U)$ have equal volumes under them (or equivalently, the two peaks in $P_{\mu, \beta}(N)$ have equal areas). Portions of the coexistence envelope sufficiently far from the critical point cannot be accurately obtained via the histograms near the critical point, and the following procedure is adopted for obtaining accurate estimates of the two coexistence volume fractions $\phi_{\text {lean }}$ and $\phi_{\text {rich. }}$ First, rough estimates of the coexistence chemical potential at, e.g., $T=0.9 T_{\mathrm{c}}$ are obtained using histograms near the critical point. Additional simulation runs are now performed around this new $T$ and $\mu$ to obtain accurate values of $\phi_{\text {lean }}$ and $\phi_{\text {rich. }}$. At the same time, the resulting histograms are combined with the existing histograms near the critical point to now estimate the coexistence $\mu$ at even lower temperatures. The procedure is repeated until the desired temperature range has been covered. More details on this procedure is available elsewhere. ${ }^{24}$

The structural properties of the branched polymers in the form of the radius of gyration of polymer chains $\left(R_{\mathrm{g}}\right)$ are also computed during the simulation runs via the relation

$$
R_{\mathrm{g}}=\left(\frac{1}{r_{i=1}^{r}}\left(\mathbf{r}_{i}-\mathbf{r}_{\mathrm{cm}}\right)\left(\mathbf{r}_{i}-\mathbf{r}_{\mathrm{cm}}\right)\right)^{1 / 2}
$$

where $\mathbf{r}_{i}$ denotes the coordinates of the polymer bead $i$, 
while $\mathbf{r}_{\mathrm{cm}}$ denotes the coordinates of the center of mass of the polymer chain.

\section{Lattice Cluster Theory}

A quite sophisticated theory for predicting the thermodynamics of polymers is the lattice cluster theory proposed by Freed and co-workers. ${ }^{12,30}$ Starting from the same lattice-based polymer-solvent system employed by Flory and Huggins, ${ }^{9}$ the LCT is based on enumerating the mixing entropy and the mixing enthalpy of a polymer solution with the aid of cluster diagrams. This leads to a more rigorous prediction for the free energy of mixing than that originally derived by Flory and Huggins. LCT is equally applicable to branched polymers as well as structured solvent molecules occupying more than a single site. According to LCT, the free energy change $(\triangle A)$ accompanying the mixing of a lattice polymer with a solvent is given by

$$
\begin{gathered}
\beta \Delta A / N_{\mathrm{T}}=\phi \ln (\phi) / r+(1-\phi) \ln (1-\phi)+ \\
a_{0} \phi(1-\phi)+a_{1} \phi^{2}(1-\phi)+a_{2} \phi^{3}(1-\phi)+ \\
A_{1} \phi(1-\phi)+\left(A_{2}+B_{3}\right) \phi^{2}(1-\phi)^{2}+A_{3} \phi^{2}(1-\phi)^{2} \times \\
(1-2 \phi)^{2}+A_{4} \phi^{2}(1-\phi)^{2}(1-6 \phi(1-\phi) \times \\
\left.\left(3 \phi^{2}-3 \phi+2\right)\right)+\left(B_{1}+B_{2}\right) \phi(1-\phi)^{2}+ \\
B_{4} \phi^{3}(1-\phi)^{2}+C_{1} \phi(1-\phi)^{2}(1-2 \phi)^{2}+C_{2} \phi(1-\phi)^{3}+ \\
C_{3} \phi^{2}(1-\phi)^{3}(1-3 \phi)+C_{4} \phi(1-\phi)^{4}(17)
\end{gathered}
$$

where $N_{\mathrm{T}}=r N_{\mathrm{p}}+N_{\mathrm{s}}$ is the total number of lattice sites available to the polymer and solvent. The first five terms on the right-hand side of eq 17 represent the athermal entropy of mixing, while the subsequent terms represent the enthalpy of mixing. The constants $a_{i}(i=0,1,2)$ and $A_{i}, B_{i}, C_{i}(i=1, \ldots, 4)$ are given by

$$
\begin{gathered}
A_{1}=\beta \epsilon z / 2 \\
A_{2}=-(\beta \epsilon)^{2} z / 4 \\
A_{3}=-(\beta \epsilon)^{3} z / 12 \\
A_{4}=-(\beta \epsilon)^{4} z / 48 \\
B_{1}=-\beta \epsilon N_{1} \\
B_{2}=\beta \epsilon(2 N(2)+N(3)+3 N(\perp)+ \\
N(12)-N(1) N(2) M) / z \\
B_{3}=-2 \beta \epsilon N(1)\left(2 N(1)+N(11)-N(1)^{2} M\right) / z \\
B_{4}=-4 \beta \epsilon N(1)^{3} / z \\
C_{1}=-(\beta \epsilon)^{2} N(1) / 2 \\
C_{2}=-(\beta \epsilon)^{2} N(2) \\
C_{3}=-(\beta \epsilon)^{2} N(1)^{2} \\
C_{4}=-(\beta \epsilon)^{2}\left(N(11)-N(1)^{2} M\right) / 2 \\
a_{0}=N(1)^{2} / z+\left[-4 N(1) N(2)+8 N(1)^{3} / 3-2 N(1) \times\right. \\
N(3)+N(2)^{2}-2 N(1)(N(12)-N(1) N(2) M)+ \\
\left.2 N(1)^{4}+2 N(1)^{2}\left(N(11)-N(1)^{2} M\right)-6 N(1) N(\perp)\right] / z^{2} \\
a_{1}=\left[8 N(1)^{3} / 3+2 N(1)^{4}+2 N(1)^{2}(N(11)-\right. \\
\left.\left.a_{2}=2 N(1)^{4} / z^{2} \quad N(1)^{2} M\right)\right] / z^{2}
\end{gathered}
$$

The terms $N(i)$ in the equation above are given by

$$
\begin{gathered}
N(i)=N_{\mathrm{i}} / M \text { for } i=1,2,3, \perp \\
N(i j)=N_{i j} / M \quad \text { for } i=1, \quad j=1,2
\end{gathered}
$$

where $N_{1}, N_{2}, N_{3}, N_{\perp}, N_{11}$, and $N_{12}$ are combinatorial terms that depend on the molecular structure of the polymer and its molecular weight $r . N_{i}(i=1,2,3)$ represents the number of $i$ sequential bonds in the polymer architecture, $N_{\perp}$ represents the number of ways in which three bonds meet at a lattice site, and $N_{11}$ represents the number of nonsequential bonds in a polymer chain ${ }^{31}$ while $N_{12}$ represents the distinct number of ways of choosing a single bond and two sequential bonds in the polymer chain. Table 1 tabulates the expressions for $N_{i}, N_{i j}$, and $N_{\perp}$ for the five different polymer architectures considered in this study in terms of the polymerization index $r$. Note that eq 17 reduces to the classic Flory-Huggins mixing free-energy when only $Q(\beta \in z)$ terms are considered.

The coexistence points are computed by equating the chemical potential of the polymer and solvent in the polymer-lean and polymer-rich phases. The chemical potentials of the polymer $\left(\mu_{\mathrm{p}}\right)$ and the solvent $\left(\mu_{\mathrm{s}}\right)$ are computed via the relationships

$$
\begin{gathered}
\Delta \mu_{\mathrm{p}}=r \frac{\Delta A}{N_{\mathrm{T}}}-r(1-\phi) \frac{\partial \Delta A / N_{\mathrm{T}}}{\partial \phi} \\
\Delta \mu_{\mathrm{s}}=\frac{\Delta A}{N_{\mathrm{T}}}-\phi \frac{\partial \Delta A / N_{\mathrm{T}}}{\partial \phi}
\end{gathered}
$$

The critical point of the polymers $\left(T_{\mathrm{c}}, \phi_{\mathrm{c}}\right)$ are evaluated via the following equations

$$
\left.\frac{\partial \Delta \mu_{\mathrm{p}}}{\partial \phi}\right|_{T_{\mathrm{c}}, \phi_{\mathrm{c}}}=0,\left.\quad \frac{\partial^{2} \Delta \mu_{\mathrm{p}}}{\partial \phi^{2}}\right|_{T_{\mathrm{c}}, \phi_{\mathrm{c}}}=0
$$

The complete set of LCT expressions for the chemical potentials and their derivatives are provided in the Appendix.

\section{Results and Discussion}

The critical parameters $\left(T_{\mathrm{c}}\right.$ and $\left.\phi_{\mathrm{c}}\right)$ obtained via Monte Carlo simulations for the polymer structures of Figure 1 and polymerization indices $(r)$ are tabulated in Table 2 . The results clearly indicate that branching has a profound effect on the critical parameters of a polymer. This dependence of $T_{\mathrm{c}}$ and $\phi_{\mathrm{c}}$ on branching may be visualized better by plotting them vs the parameter $N_{\mathrm{b}}$ which characterizes the degree of branching of a polymer. The quantity $N_{\mathrm{b}}$ denotes the total number of branches present in a polymer, inclusive of the first and second generation branches. $N_{\mathrm{b}}$ is therefore equal to 0 , $1,2,4$, and 6 for the polymers structures of the type L, B1, B2, B4, and D of Figure 1, respectively.

Figure 2 shows that an increase in the degree of branching causes a lowering of $T_{\mathrm{c}}$ for polymers of all molecular weights $r$. Intuitively, the drop in $T_{\mathrm{c}}$ with branching occurs as a result of the polymer chains becoming more compact as the degree of branching increases (see Table 2 for values of the mean radii of gyration $R_{\mathrm{g}}$ of the polymers chains computed from the Monte Carlo simulations at the critical point), which decreases the amount of enthalpic interactions between neighboring polymer chains. A lower temperature is 
Table 1. Combinatorial Coefficients for the Five Different Polymer Architectures Considered in This Study

\begin{tabular}{llllll}
\hline & \multicolumn{1}{c}{$\mathrm{L}$} & \multicolumn{1}{c}{$\mathrm{B} 1$} & \multicolumn{1}{c}{$\mathrm{B} 2$} & \multicolumn{1}{c}{$\mathrm{B} 4$} \\
\hline$N_{1}$ & $r-1$ & $r-1$ & $r-1$ & $r-1$ & $r-1$ \\
$N_{2}$ & $r-2$ & $r-1$ & $r+1$ & $r+4$ & $r+5$ \\
$N_{3}$ & $r-3$ & $r-1^{a}$ & $r+3^{a}$ & $r+9^{a}$ & $r+3^{a}$ \\
$N_{\perp}$ & 0 & 1 & 4 & 8 & 8 \\
$N_{11}$ & $(r-2)(r-3)$ & $(r-1)(r-4)$ & $r(r-4)$ & $(r+1)(r-6)$ & $r^{2}-5 r-8$ \\
$N_{12}$ & $(r-3)(r-4)$ & $r^{2}-6 r+2^{a}$ & $(r-7)(r+3)^{a}$ & $r^{2}-r-54^{a}$ & $r^{2}-61^{a}$
\end{tabular}

${ }^{a}$ Formula applicable when the polymerization index of each polymer branch is larger than 1 .

Table 2. Critical Point Data via Monte Carlo Simulations and Lattice Cluster Theory for the Different Polymer Structures $^{a}$

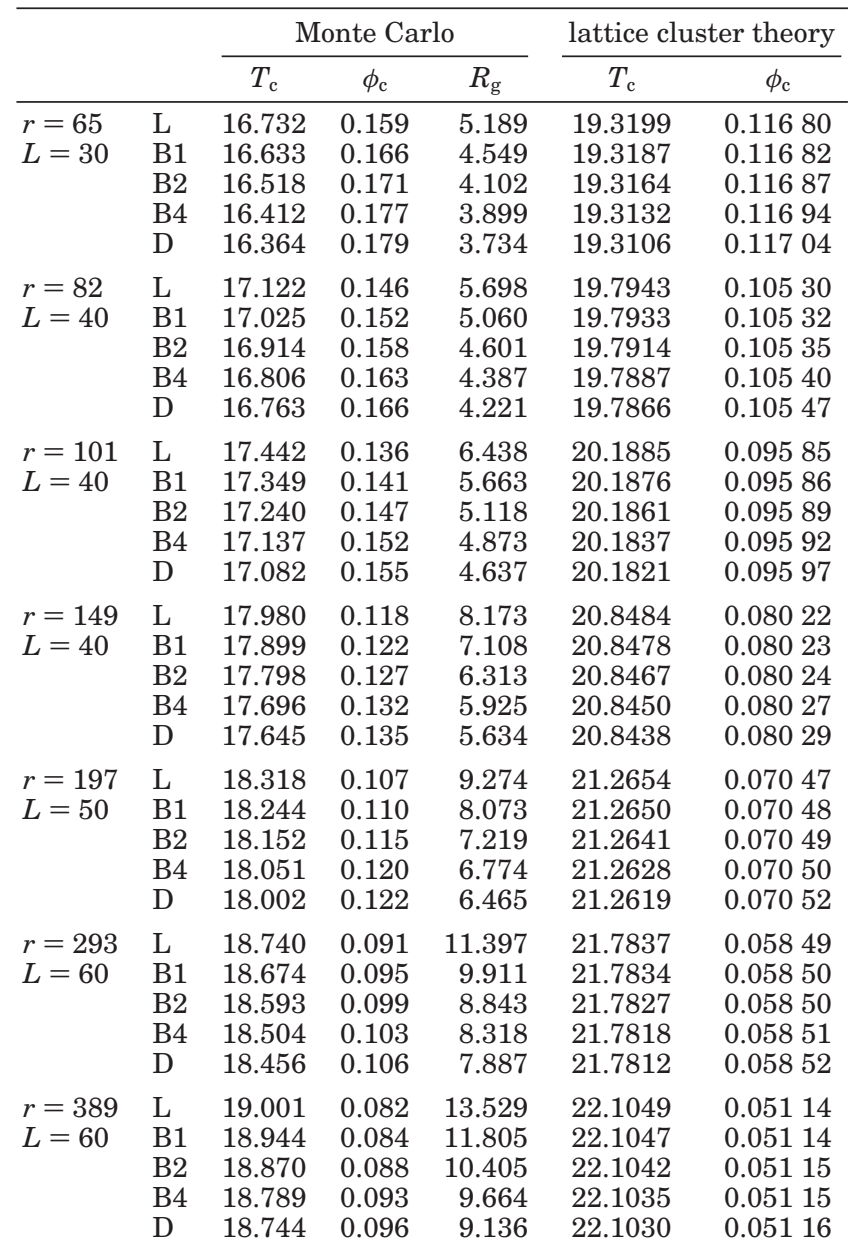

${ }^{a}$ Also tabulated are the simulation box lengths and the radii of gyration of polymer chains obtained from Monte Carlo simulations at the critical point. Statistical uncertainties in the simulation results are smaller than 0.005 in the case of $T_{\mathrm{c}}$ and $R_{\mathrm{g}}$, and smaller than 0.002 in the case of $\phi_{\mathrm{c}}$

therefore required to "coerce" the polymer solution to phase separate. The relative magnitude of the drop in $T_{\mathrm{c}}$ with $N_{\mathrm{b}}$ characterized by $\left(\left(1-T_{\mathrm{c}}\right) /\left.T_{\mathrm{c}}\right|_{N_{\mathrm{b}}=0}\right)$ is about $2 \%$ for the most branched polymer architecture and shortest chain considered in this study ( $\mathrm{D} ; r=65)$. Longer chains display smaller drops in $T_{\mathrm{c}}$ with respect to the degree of branching i.e., the branched polymers increasingly behave like linear polymers at large polymerization indices. The above observations are consistent with experiments of Alessi et al. ${ }^{8}$ who observed that the critical temperatures of branched (star-shaped with 8 arms) polystyrene (PS) in methylcyclohexane (MCH) solvent were 5-15 K lower than that for linear polystyrene of the same molecular weight in the same solvent, and the difference decreased as the molecular weight of the polymer increased.

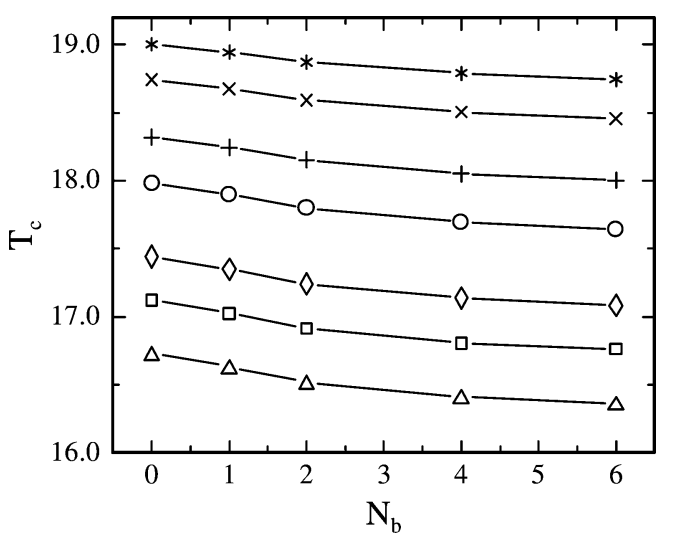

Figure 2. Dependence of critical temperature on polymer architecture for different polymerization indices. The symbols represent results for $r=65(\triangle), r=82(\square), r=101(\diamond), r=$ $149(\bigcirc), r=197(+), r=293(\times)$, and $r=389(*)$. Error bars are smaller than symbol size. The lines are meant to guide the eye.

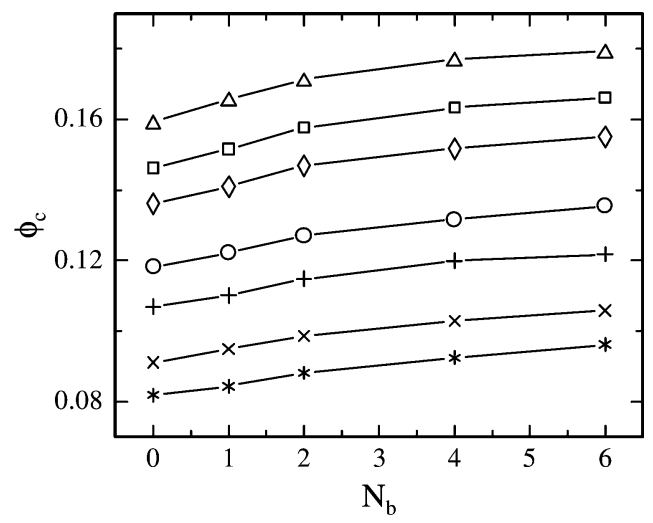

Figure 3. Dependence of critical polymer volume fraction on polymer architecture for different polymerization indices. The symbolic representation is the same as in Figure 2.

An increase in the degree of branching also causes the polymer solution to exhibit a higher $\phi_{\mathrm{c}}$ (see Figure 3 ), as predicted theoretically also. ${ }^{11}$ Again, this trend is consistent with the argument that branching generally results in more compact polymer conformations. Generally speaking, a dilute homogeneous polymer solution which is gradually being concentrated, begins to phase separate when the polymers reach a concentration where they begin to significantly interpenetrate with each other. This concentration is typically known as the polymer overlap concentration $\phi^{*}$, approximately given by

$$
\phi^{*} \simeq 3 r / 4 \pi R_{\mathrm{g}}^{3}
$$

Roughly speaking, $\phi^{*}$ is of the same order of magnitude as $\phi_{c}$. This means that the more compact branched polymers need to be present at higher concentrations than their more extended linear counterparts in order 


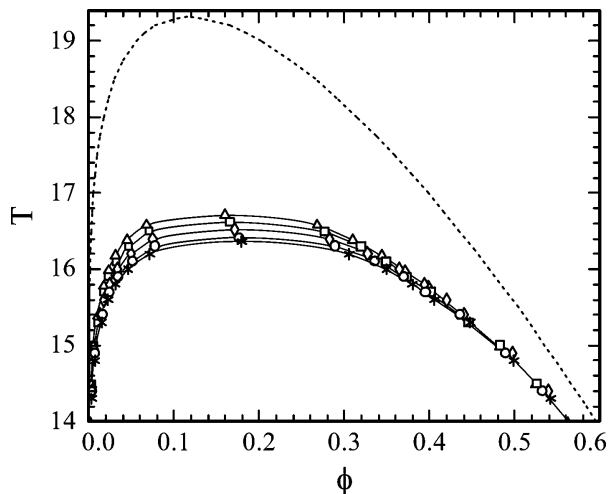

Figure 4. Phase coexistence envelopes for the five different polymer architectures at $r=65$. The symbols represent Monte Carlo results for L $(\triangle), \mathrm{B} 1(\square), \mathrm{B} 2(\diamond), \mathrm{B} 4(\mathrm{O})$, and D (*) type polymers; the solid lines passing through the symbols are guides to the eye. The dotted line represents the phase coexistence envelopes obtained from the lattice cluster theory. The error bars in the coexistence temperatures and volume fractions are smaller than size of the symbols.

to interact at the same degree with each other as the linear polymers do. Hence, branched polymers begin to phase separate at higher polymer concentrations than the polymer concentrations required by linear polymers to phase separate. The above predictions are in agreement with the experimental results of Yokoyama et al., ${ }^{6}$ who observed that $\phi_{\mathrm{c}}$ was equal to 0.03 and 0.04 for linear and 6.3-arm PS in cyclohexane solvent, respectively. Alessi et al., ${ }^{8}$ on the other hand, do not show any noticeable differences in the $\phi_{c}$ of star-shaped and linear versions of PS in $\mathrm{MCH}$ solvent. Clearly, more experiments are needed to fully confirm our prediction. A direct application of eq 23 however overestimates the magnitude of rise in $\phi_{\mathrm{c}}$ with the degree of branching. According to the same argument, branched polymers should also exhibit a higher miscibility than linear polymers at a given temperature $T<T_{\mathrm{c}}$-the polymer miscibility being given by the polymer volume fraction on the low polymer density part of the coexistence envelope (binodal). Indeed, in Figure 4, the low-density phase coexistence envelopes for a $r=65$ polymer chain are observed to shift rightwards (and downward) as the degree of branching increases in Figure 4, thus confirming an increased polymer miscibility with branching. Such trends in the polymer miscibility vs $N_{\mathrm{b}}$ have not been tested experimentally so far.

Table 2 tabulates the critical parameters from LCT alongside those obtained via simulations, while Figure 4 plots the phase coexistence envelopes for the $r=65$ isomers obtained from both the LCT and simulations. Several key observations may be made. First, the LCT consistently overpredicts the value of $T_{\mathrm{c}}$ and underpredicts the value of $\phi_{c}$ as compared to simulations. This overestimation of $T_{\mathrm{c}}$ by the LCT is a characteristic feature of all mean field theories due to their neglect of fluctuations. ${ }^{32}$ There is also an analogous overestimation of the order-disorder transition temperature $T_{\text {odt }}$ in block copolymers by the selfconsistent field theory of Leibler ${ }^{33}$ when compared to Fredrickson and Helfand's theory, ${ }^{34}$ which includes the effect of fluctuations. ${ }^{35}$ Nonetheless, the LCT performs better than the Flory-Huggins theory for linear polymers. For instance, the Flory-Huggins theory predicts $T_{\mathrm{c}}=20.579$ for a $r=65$ linear polymer, a worse prediction than that by the LCT when compared to Monte Carlo results.

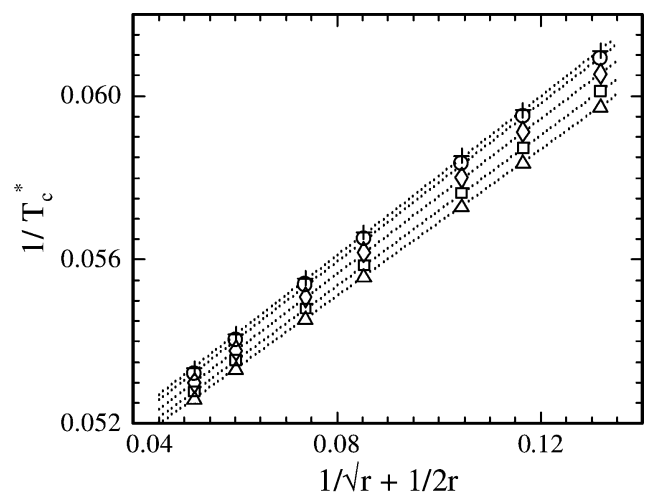

Figure 5. Inverse critical temperature vs Flory-Shultz parameter for different polymer architectures. The symbols represent Monte Carlo simulation results: $\mathrm{L}(\triangle), \mathrm{B} 1(\square), \mathrm{B} 2$ $(\diamond)$, B4 $(\bigcirc)$, and D $(+)$. Error bars are smaller than symbol size. The dotted lines represent linear fits to the simulation data.

Second, even though the LCT predicts a similar trend in $T_{\mathrm{c}}$ and $\phi_{\mathrm{c}}$ with branching as the Monte Carlo simulations, the magnitude of the drops (rises) in $T_{\mathrm{c}}\left(\phi_{\mathrm{c}}\right)$ with branching are 1-2 orders of magnitude smaller than those predicted by the Monte Carlo simulations. One possible reason for this discrepancy may be that the LCT does not properly take into account the differences in the conformation of polymers with varying degree of branching. The LCT theory severely underpredicts the changes in the radii of gyration of polymers with branching, a key factor governing the critical behavior of polymers. Another possible reason for the discrepancy may be the difference in treatment of fluctuations in the LCT and simulations. We are unaware of any theory through which we can evaluate the impact of fluctuations on the phase behavior of branched polymers. Several other studies in the past have employed the LCT to evaluate the role of branching on the critical properties of polymers. ${ }^{36,37}$ However, these studies only considered highly branched polymers (dendrimers with large generation numbers) for their analysis, where they naturally observed much larger deviations in $T_{\mathrm{c}}$ than those observed in the current study (see, for example, Figure 15 of ref 36 ).

A comparison of the phase coexistence envelopes obtained via Monte Carlo simulations with those predicted by LCT shows further disagreement between the two sets of results. The phase coexistence envelopes predicted by LCT are much narrower (larger curvature) near the critical point than those obtained via MC simulations. Additionally, the LCT phase coexistence envelopes for the different polymer architectures show no noticeable change in their shape and location unlike those obtained via MC simulations which shrink in the $\phi$ direction and shift downward in the $T$ direction. The agreement between the LCT and MC phase coexistence envelopes however gets better at higher polymer volume fractions, the regime where the mean field approximation of the LCT becomes more valid.

In Figure 5, we have tested the results for $T_{\mathrm{c}}$ of the branched polymers against the Shultz-Flory relationship $^{38}$ given by

$$
\frac{1}{T_{\mathrm{c}}(r)}-\frac{1}{T_{\mathrm{c}}(\infty)} \propto \frac{1}{\sqrt{r}}+\frac{1}{2 r}
$$

Clearly, the branched polymers (like their linear counterparts) seem to follow the Shultz-Flory relationship. 
The slopes for the branched polymers $(\approx 0.09)$ are very similar to those corresponding to the linear polymers. ${ }^{39}$ Extrapolation of these data to infinite chain lengths gives $T_{\mathrm{c}}(\infty)=20.84$ for the linear polymers $(\mathrm{L})$ and $T_{\mathrm{c}}(\infty)=20.82,20.79,20.73$, and 20.68 for branched polymers of type B1, B2, B4, and D, respectively. It has been shown that this extrapolated infinite chain length critical temperature is equal to the $\Theta$ temperature of the polymers, $\Theta$, where the enthalpic attractions between the polymer segments are exactly balanced by the excluded volume repulsion (entropic) between these segments, or when the second virial term in the osmotic pressure becomes zero. ${ }^{29,40}$ Given the equivalence of $T_{\mathrm{c}}(\infty)$ and $\Theta$, our simulation results suggest that $\Theta$ decreases minimally with the degree of branching, in agreement with predictions by the mean field theory of Candau et al. ${ }^{41}$

The critical volume fractions obtained from our simulations when plotted in a log- $\log$ fashion with respect to the chain lengths $r$ suggest that $\phi_{\mathrm{c}}$ follows a powerlaw scaling with respect to the chain lengths both for linear as well as branched polymers, as given by

$$
\phi_{\mathrm{c}} \propto r^{-x_{2}}
$$

with the coefficient $x_{2} \approx 0.37$ for branched and linear polymers alike. It has been speculated that very long chain lengths will eventually raise this coefficient toward its theoretical value of $1 / 2 .{ }^{42,43}$ To the best of our knowledge, the present study is the first to show that moderately branched polymers follow similar scaling relations for $T_{\mathrm{c}}$ and $\phi_{\mathrm{c}}$ as linear polymers. It would be interesting to investigate in the future if the above scaling relations hold for highly branched polymers as well.

Finally, the scaling behavior (with respect to chain length) of the mean radii of gyration of the simulated linear and branched polymers both at the $\Theta$ temperature and in the athermal limit $(T \rightarrow \infty)$ have been obtained. The radii of gyration were computed from the Monte Carlo simulations using eq 16 in the dilute limit $\phi \ll \phi^{*}$, where the overlap concentration was computed via eq 23. Additionally, a large temperature of $T(=500)$ $\gg T_{\mathrm{c}}$ was employed for computing $R_{\mathrm{g}}$ in the athermal limit, while a temperature of $T=T_{\mathrm{c}}(\infty)$ was employed for the $\Theta$ point simulations (hence a different $T$ for each polymer architecture). In Figure 7 , we have plotted $R_{\mathrm{g}}$ corresponding to the five polymer architectures in the two limits described above. $R_{\mathrm{g}}$ is expected to follow a power-law scaling behavior with respect to $r$ for all polymer architectures as given by

$$
R_{\mathrm{g}} \propto r^{v}
$$

where $v$ is a scaling exponent. In the athermal limit, we have obtained that $v=0.58 \pm 0.01,0.58 \pm 0.01,0.58$ $\pm 0.01,0.57 \pm 0.01$, and $0.57 \pm 0.01$ for our L, B1, B2, B4, and D polymer architectures. Hence, both the linear and branched polymers exhibit scaling exponents close to the theoretical value of $v=0.588 .{ }^{44}$ On the other hand, at the $\Theta$ point, we obtain $v=0.51 \pm 0.01,0.51 \pm$ $0.01,0.51 \pm 0.01,0.50 \pm 0.01$, and $0.50 \pm 0.01$ for the L, B1, B2, B4, and D polymer architectures. Again, this is in close agreement with the theoretical prediction of $v=0.5$ for chains exhibiting prefectly random walks. Given the fact that self-avoiding polymers chains begin to behave like random chains at temperatures close to

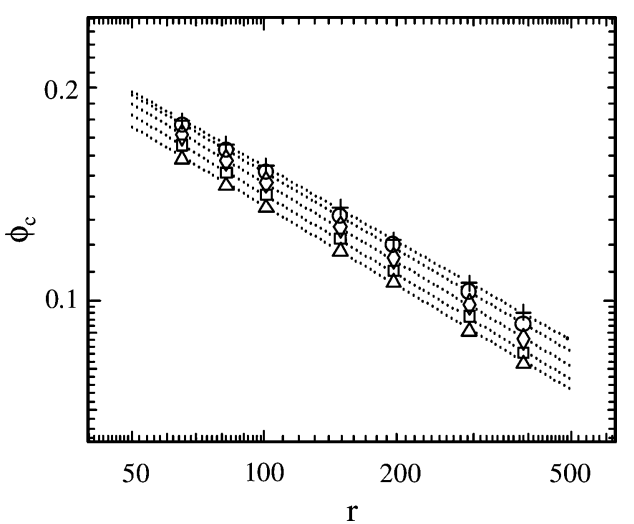

Figure 6. Critical polymer volume fraction $(\phi)$ vs polymerization index $(r)$ for different polymer architectures in a log$\log$ plot. The symbols represent Monte Carlo simulation results: $\mathrm{L}(\triangle), \mathrm{B} 1(\square), \mathrm{B} 2(\diamond), \mathrm{B} 4(\bigcirc)$, and D $(+)$. Error bars are smaller than symbol size. The dotted lines indicate the power-law relationship between $\phi$ and $r$ for five polymer architectures.

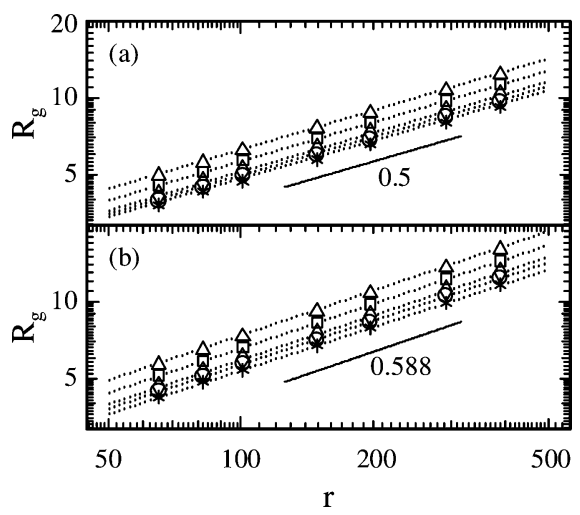

Figure 7. Radius of gyration vs polymerization index in the (a) $\Theta$ temperature limit, and (b) the athermal limit for the five polymer architectures: $\mathrm{L}(\triangle), \mathrm{B} 1(\square), \mathrm{B} 2(\diamond), \mathrm{B} 4(\bigcirc)$, and $\mathrm{D}(*)$. Error bars are smaller than symbol size.

the $\Theta$ point confirms our presumption of $T_{\mathrm{c}}(\infty)=\Theta$ for all polymer architectures.

\section{Conclusions}

The primary objective of this study has been to examine the effect of branching on the phase behavior and critical properties of polymers. Using grand canonical Monte Carlo simulations along with multihistogram reweighting and finite-size scaling analysis, we were able to accurately determine the phase coexistence envelopes and critical points for polymers of varying degree of branching and molecular weights. Our analysis shows that an increase in branching of polymers leads to a decrease in the critical temperature and an increase in the critical polymer volume fraction. We associate these trends to the simultaneous contraction of the polymer with the degree of branching. In addition, we have also carried out an exhaustive comparison of our simulation results with those obtained using the lattice cluster theory. It has been observed that even though the lattice cluster theory qualitatively yields similar trends for the variation of $T_{\mathrm{c}}$ and $\phi_{\mathrm{c}}$ with the degree of polymer branching polymers, the theory severely underestimates the magnitude of these trends. We have noted that branched polymers follow the Shultz-Flory relation similarly to the linear polymers. Our branched polymers also follow the same power-law 
behavior, with respect to the chain length, as observed in the case of linear polymers.

Currently, we have only examined moderately branched polymers. It would be interesting to examine the effect of hyperbranching such as in the case of third and fourth generation dendrimers. It would also be fruitful for researcher to examine the effect of branching along the lines of renormalization theories which can capture the effect of fluctuations directly. A more thorough analysis in the future concerning the reasons for the observed discrepancies between the Monte Carlo and lattice cluster theory will also be quite useful.

Acknowledgment. This work was supported by ACS-PRF (Grant 38165-AC9) and the Department of Energy, Office of Basic Energy Sciences (Grant DE-FG0201ER15121). We also acknowledge discussions with Prof. Karl Freed and Dr. Leo Lue.

\section{Appendix}

The chemical potential of the polymer and solvent, according to the lattice cluster theory, are given by

$$
\begin{gathered}
\beta \Delta \mu_{\mathrm{p}}=r\left[\ln (\phi) / r-(1-1 / r)(1-\phi)+a_{0}(1-\phi)^{2}+\right. \\
2 a_{1} \phi(1-\phi)^{2}+3 a_{2} \phi^{2}(1-\phi)^{2}+A_{1}(1-\phi)^{2}+ \\
\left(A_{2}+B_{3}\right) \phi(1-\phi)^{2}(2-3 \phi)+A_{3} \phi(1-\phi)^{2}(1- \\
2 \phi)\left(2-11 \phi+10 \phi^{2}\right)+A_{4} \phi(1-\phi)^{2}(2-39 \phi+ \\
\left.168 \phi^{2}-330 \phi^{3}+324 \phi^{4}-126 \phi^{5}\right)+\left(B_{1}+B_{2}\right)(1- \\
2 \phi)(1-\phi)^{2}+B_{4} \phi^{2}(1-\phi)^{2}(3-4 \phi)+C_{1}(1-\phi)^{2}(1- \\
2 \phi)\left(1-8 \phi+8 \phi^{2}\right)+C_{2}(1-\phi)^{3}(1-3 \phi)+C_{3} \phi(1- \\
\left.\phi)^{3}(1-5 \phi)(2-3 \phi)+C_{4}(1-\phi)^{4}(1-4 \phi)\right](27) \\
\beta \Delta \mu_{\mathrm{s}}=\ln (1-\phi)+(1-1 / r) \phi+a_{0} \phi^{2}-a_{1} \phi^{2}(1- \\
2 \phi)-a_{2} \phi^{3}(2-3 \phi)+A_{1} \phi^{2}-\left(A_{2}+B_{3}\right) \phi^{2}(1-\phi)(1- \\
3 \phi)-A_{3} \phi^{2}(1-\phi)(1-2 \phi) \times\left(1-9 \phi+10 \phi^{2}\right)- \\
A_{4} \phi^{2}(1-\phi)\left(1-27 \phi+138 \phi^{2}-294 \phi^{3}+306 \phi^{4}-\right. \\
\left.126 \phi^{5}\right)+2\left(B_{1}+B_{2}\right) \phi^{2}(1-\phi)-2 B_{4} \phi^{3}(1-\phi)(1- \\
2 \phi)+2 C_{1} \phi^{2}(1-\phi)(1-2 \phi)(3-4 \phi)+3 C_{2} \phi^{2}(1- \\
\phi)^{2}-C_{3} \phi^{2}(1-\phi)^{2}\left(1-10 \phi+15 \phi^{2}\right)+4 C_{4} \phi^{2}(1-\phi)^{3}
\end{gathered}
$$

while the first and second derivatives of the polymer chemical potential are given by

$$
\begin{gathered}
\beta \frac{\partial \Delta \mu_{\mathrm{p}}}{\partial \phi}=r\left[1-(1-1 / \phi) / r-2 a_{0}(1-\phi)+2 a_{1}\left(3 \phi^{2}-\right.\right. \\
4 \phi+1)+3 a_{2}\left(4 \phi^{3}-6 \phi^{2}+2 \phi\right)+2 A_{1}(\phi-1)+ \\
\left(A_{2}+B_{3}\right)\left(2-14 \phi+24 \phi^{2}-12 \phi^{3}\right)+A_{3}(2-38 \phi+ \\
\left.192 \phi^{2}-396 \phi^{3}+360 \phi^{4}-120 \phi^{5}\right)+2 A_{4}(1-43 \phi+ \\
372 \phi^{2}-1410 \phi^{3}+2880 \phi^{4}-3312 \phi^{5}+2016 \phi^{6}- \\
\left.504 \phi^{7}\right)+2\left(B_{1}+B_{2}\right)(1-\phi)(3 \phi-2)+2 B_{4} \phi \times \\
(\phi-1)\left(-3+12 \phi-10 \phi^{2}\right)+2 C_{1}(1-\phi)(-6+39 \phi- \\
\left.72 \phi^{2}+40 \phi^{3}\right)+6 C_{2}(\phi-1)^{2}(2 \phi-1)+2 C_{3}(\phi-1)^{2} \\
\left.\left(1-17 \phi+55 \phi^{2}-45 \phi^{3}\right)+4 C_{4}(2-5 \phi)(-1+\phi)^{3}\right]
\end{gathered}
$$

$$
\begin{gathered}
\beta \frac{\partial^{2} \Delta \mu_{\mathrm{p}}}{\partial \phi^{2}}=r\left[2 a_{0}+4 a_{1}(3 \phi-2)+3 a_{2}\left(12 \phi^{2}-12 \phi+\right.\right. \\
2)+2 A_{1}+\left(A_{2}+B_{3}\right)\left(-14+48 \phi-36 \phi^{2}\right)+ \\
A_{3}\left(-38+384 \phi-1188 \phi^{2}+1440 \phi^{3}-600 \phi^{4}\right)+2 A_{4} \\
\left(-43+744 \phi-4230 \phi^{2}+11520 \phi^{3}-16560 \phi^{4}+\right. \\
\left.12096 \phi^{5}-3528 \phi^{6}\right)+2\left(B_{1}+B_{2}\right)(5-6 \phi)+ \\
2 B_{4}\left(3-30 \phi+66 \phi^{2}-40 \phi^{3}\right)+2 C_{1}(4 \phi-3)(-15+ \\
\left.54 \phi-40 \phi^{2}\right)+12 C_{2}(\phi-1)(3 \phi-2)+2 C_{3}(1- \\
\phi)\left(-19+161 \phi-355 \phi^{2}+225 \phi^{3}\right)+4 C_{4}(11- \\
\left.20 \phi)(\phi-1)^{2}-1 / r \phi^{2}\right]
\end{gathered}
$$

\section{References and Notes}

(1) Tomalia, D. A.; Dvornic, P. R. Nature (London) 1994, 372, $617-618$.

(2) Landskron, K.; Ozin, G. A. Science 2004, 306, 1529-1532.

(3) Esfand, R.; Tomalia, D. A. Drug Discov. Today 2001, 6, 427436.

(4) Halford, B. Chem. Eng. News 2005, 83 (24), 30.

(5) Freire, J. J. Adv. Polym. Sci. 1999, 143, 35-112.

(6) Yokoyama, H.; Takano, A.; Okado, M.; Nose, T. Polymer 1991 $32,3218-3224$.

(7) Terao, K.; Okumoto, M.; Nakamura, Y.; Norisuye, T.; Teramoto, T. Macromolecules 1998, 31, 6885-6891.

(8) Alessi, M. L.; Bittner, K. C.; Greer, S. C. J. Polym. Sci. 2004 $42,129-145$.

(9) Flory, P. J. Principles of Polymer Chemistry; Cornell University Press: Ithaca, NY, 1979

(10) Daoud, M.; Pincus, P.; Stockmayer, W. H.; Witten, T. Macromolecules 1983, 16, 1833-1839.

(11) Saeki, S. Polymer 2000, 41, 8331-8338.

(12) Nemirovsky, A. M.; Bawendi, M. G.; Freed, K. F. J. Chem. Phys. 1987, 87, 7272-7284.

(13) Mazur, J.; McCrackin, F. Macromolecules 1977, 10, 326-332.

(14) Sikorski, A. Polymer 1993, 34, 1271-1281.

(15) Hsu, H. P.; Nadler, W.; Grassberger, P. Macromolecules 2004 $37,4658-4663$.

(16) Grest, G. S.; Fetters, L. J.; Huang, J. S.; Richter, D. Adv. Chem. Phys. 1996, 94, 67-163.

(17) Maury-Evertsz, J. R.; Estevez, L. A.; Lopez, G. E. J. Chem. Phys. 2004, 121, 8652-8657.

(18) Zifferer, G. Macromol. Theor. Simul. 1999, 8, 433-462.

(19) Potoff, J. J.; Siepmann, J. I. Phys. Rev. Lett. 2000, 85, 34603463.

(20) Nath, S. K.; de Pablo, J. J. Mol. Phys. 2000, 98, 231-238.

(21) Frenkel, D.; Mooij, G. C. A. M.; Smit, B. J. Phys.: Condens. Matter 1992, 4, 3053-3076.

(22) Rosenbluth, M. N.; Rosenbluth, A. W. J. Chem. Phys. 1955 , $23,356-359$.

(23) Frenkel, D.; Smit, B. Understanding Molecular Simulations; Academic Press: London, 1996.

(24) Panagiotopoulos, A. Z. J. Phys.: Condens. Matter 2000, 12, R25-R52.

(25) Ferrenberg, A. M.; Swendsen, R. H. Phys. Rev. Lett. 1988, $61,2635-2638$

(26) Ferrenberg, A. M.; Swendsen, R. H. Phys. Rev. Lett. 1989, $63,1195-1198$.

(27) Wilding, N. B.; Bruce, A. D. J. Phys.: Condens. Matter 1992, 4, 3087-3108

(28) Wilding, N. B. Phys. Rev. E 1995, 52, 602-611.

(29) Panagiotopoulos, A. Z.; Wong, V.; Floriano, M. A. Macromolecules 1998, 31, 912-918.

(30) Dudowicz, J.; Freed, K. F.; Madden, W. G. Macromolecules 1990, 23, 4803-4819.

(31) We would like to point out that, in several previous papers, ${ }^{36,37} N_{11}$ has been defined as the number of distinct ways of choosing two nonsequential bonds in the polymer chain, but the authors did not incorporate the remaining factor of 2 ! within the formula for the mixing free energy.

(32) Mackie, A. D.; Panagiotopoulos, A. Z.; Kumar, S. K. J. Chem. Phys. 1994, 102, 1014-1023.

(33) Leibler, L. Macromolecules 1980, 13, 1602-1617. 
(34) Fredrickson, G. H.; Helfand, E. J. Chem. Phys. 1987, 87, 697705.

(35) Bates, F. S.; Rosedale, J. H.; Fredrickson, G. H. J. Chem. Phys. 1990, 92, 6255-6270.

(36) Lue L.; Prausnitz, J. M. Macromolecules 1997, 30, 66506657.

(37) Jang, J. G.; Bae, Y. C. Polymer 1999, 40, 6761-6768.

(38) Shultz, A. R.; Flory, P. J. J. Am. Chem. Soc. 1952, 74, 47604767.

(39) Hehmeyer, O. J.; Arya, G.; Panagiotopoulos, A. Z. J. Phys. Chem. B 2004, 108, 6809-6815.
(40) Sheng, Y.-J.; Panagiotopoulos, A. Z.; Kumar, S. K.; Szleifer, I. Macromolecules 1994, 27, 400-406.

(41) Candau, F.; Rempp, P.; Benoit, H. Macromolecules 1972, 5, $627-635$.

(42) Frauenkron, H.; Grassberger, P. J. Chem. Phys. 1997, 107, 9599-9608.

(43) Yan, Q.; dePablo, J. J. J. Chem. Phys. 2000, 113, 5954-5957.

(44) de Gennes, P. G. Scaling Concepts in Polymer Physics; Cornell University Press: Ithaca, NY, 1979.

MA0515376 